\title{
Augmentation of Pulmonary Surfactant Secretion by Lung Expansion at Birth
}

\author{
EDWARD E. LAWSON, ${ }^{(33)}$ ROBYN L. BIRDWELL, PEARL S. HUANG, AND \\ H. WILLIAM TAEUSCH, JR. \\ Children's Hospital Medical Center and the Department of Pediatrics, Harvard Medical School, Boston, \\ Massachusetts, USA
}

\begin{abstract}
Summary
To study the effect of lung expansion at birth on surfactant secretion, we delivered by hysterotomy 11 litters of rabbit pups at 30 days of gestation and divided them into three groups that were killed: 1) after $\mathbf{3 0} \mathrm{min}$ air-breathing, 2) after $\mathbf{3 0} \mathrm{min}$ nitrogenbreathing, and 3) after 30 min tracheal occlusion. Each group was compared to a littermate group killed at birth. Groups 2) and 3) continued respiratory efforts for $\mathbf{3 0} \mathrm{min}$ despite progressive asphyxia. Six additional litters were pretreated with atropine; at delivery one-half of each litter was killed, the remaining pups were subjected to $\mathbf{3 0} \mathrm{min}$ of hypoxic gas breathing. After sacrifice, alveolar surfactant was recovered by saline lavage and estimated quantitatively on a surface-tension balance. Surfactant concentration at birth was $1.40 \pm 0.22 \mathrm{mg} / \mathrm{g}$ dry lung and increased to 1.86 $\pm 0.19( \pm$ SEM) after $30 \mathrm{~min}$ air-breathing $(P<0.01)$. Also, surfactant was increased in the nitrogen-breathing pups (from 1.61 \pm 0.35 in littermate controls to $2.41 \pm 0.58 ; P<0.03$, but not to a significant degree in the occluded group $(1.34 \pm 0.33$ vs. $1.41 \pm$ $0.28)$, or the atropine pretreated breathing pups $(1.77 \pm 0.29$ vs. $1.89 \pm 0.25$ ).
\end{abstract}

\section{Speculation}

The data indicate that lung expansion at birth enhances surfactant release from intracellular sites and suggest that the vagus nerve mediates this effect. It is possible that the effect of maternal atropine may have clinical significance in preterm infants.

Studies with newborn rabbits have shown that the percent of acetone precipitable lecithin in pulmonary lavage (12), pulmonary stability (29), and pulmonary lavage phospholipid content (25) increase after birth. These findings indicate that immediately after birth the secretion of surfactant into the alveoli is increased over and above the fetal secretion rate. With the onset of breathing, augmented surfactant secretion may be due to mechanical, neuronal, or humoral factors. Both lower surface tension and increased saturated lecithin in alveolar wash result from constant air-expansion of excised adult dog lungs (8), a mechanical stimulus. In rabbits, fetal injection of pilocarpine or $\beta$-adrenergic stimulants (e.g., isoxsuprine) also result in increased alveolar surfactant $(5,6,7,18,30)$. These pharmacologic effects suggest autonomic control of surfactant secretion.

To determine the physiologic stimulus for increased alveolar surfactant after birth, we measured quantitatively the surfaceactive material (SAM) recovered by pulmonary lavage of newborn. rabbit pups. Four groups of rabbits were studied: one group was allowed to air-breathe after birth; the second was subjected to a nitrogen environment; the third to tracheal occlusion from birth; and the fourth was pretreated with atropine and subjected to hypoxia after delivery. Asphyxia is a potent stimulus leading to increased endogenous catecholamine levels (4). The nitrogen- breathing and the occluded pups differentiate the effects of asphyxia with and without gas ventilation of the lungs. The findings in the atropine pretreated groups establish that neural factors mediate surfactant secretion at birth.

\section{MATERIALS AND METHODS}

\section{ANIMAL PROCEDURES}

Twenty litters of New Zealand white rabbits were used. The gestation of each litter was known to be 30 days within $3 \mathrm{hr}$ (term $=31$ days). In 11 litters, the does were sacrificed immediately before laparotomy with an iv infusion of $300 \mathrm{mg}$ pentobarbital. Within 2 min, all the pups of a litter were gently delivered within their amniotic sacs by hysterotomy. The pups of each litter were divided into pairs. One pair, designated as control, was immediately killed with $30 \mathrm{mg}$ ip pentobarbital having never breathed air. The second pair was allowed to breathe air under a warming light. When possible, a third and fourth pair were designated. In the third pairs (from 6 of the 11 litters), asphyxia was produced by having the two pups breathe warm humidified $100 \%$ nitrogen. In the fourth pairs, gas entry was prevented by a ligature placed at delivery around the neck of each pup ( 7 of the 11 litters; designated tracheal occlusion). The occluded pups were placed under warming lights with the air-breathing pups. The occluded pups spontaneously initiated gasping respiratory efforts, but never aroused or struggled. The air-breathing, nitrogen-breathing, and tracheal occlusion pups were killed 30 min after delivery with $30 \mathrm{mg}$ pentobarbital. After the pups were killed, the trachea was cannulated with a polyethylene catheter (Intramedic 7421, Clay Adams).

At 30 days of gestation, 9 additional pregnant does were pretreated with either atropine ( 6 does) or normal saline ( 3 does) over a $1 / 2 \mathrm{hr}$ period immediately before killing. Because female rabbits have a high level of atropinesterase (26) repeated doses of atropine were given over the 30 -min-period (total, $2.4 \mathrm{mg}$ iv). This was sufficient to obliterate the pupillary light reflex. As above, the pups were delivered by hysterotomy and divided into two groups. One group was killed at birth and, to maximize ventilation, the other subjected to a 100\% nitrogen and/or 10-15\% oxygen environment for $30 \mathrm{~min}$. The pups of the three saline pretreated does were also divided into a nonbreathing control group and a group subjected to $10-15 \%$ oxygen. Because saline pretreatment did not affect the results, the data from the saline pretreated pups were combined with those of the nonpretreated nitrogen-breathing pups to contrast with the results of the atropine pretreated pups.

\section{SURFACTANT RECOVERY}

After killing, the lungs of each pup were inflated with air at 30 $\mathrm{cm}$ water pressure until lung volume stabilized; the lungs were then deflated to atmospheric pressure. Alveolar surfactant was recovered by five pulmonary saline lavages of $2 \mathrm{ml}$ each (12). Return lavage volume was not different between groups and 
averaged approximately $90 \%$. The lavage fluid of each pair of pups was pooled and chilled in ice. Any animals with gross pulmonary hemorrhage or in whom the recovery of lavage fluid was less than $70 \%$ were prospectively discarded. The lavage fluid was initially centrifuged at $400 \mathrm{~g}$ for $5 \mathrm{~min}$ to remove cellular debris. The supernatant was then frozen at $-40^{\circ}$ for 1 day-2 wk. Next, the acellular tracheal fluid was centrifuged at $78,000 \mathrm{~g}$ for 2 hr at $4^{\circ}$ to concentrate the surfactant. The supernatant was discarded and the pellet was suspended in $67 \%$ isopropyl alcohol (3).

\section{QUANTITATIVE SURFACE-TENSION MEASUREMENT}

A quantitative estimate of the lavage SAM content was made by applying duplicate aliquots of the alcoholic solution to a modified Wilhelmy surface balance of the type described by Clements et al. (3). The trough of the surface balance measured $118.2 \mathrm{~cm} \times 2.9 \mathrm{~cm} \times 1.0 \mathrm{~cm}$. Normal saline at room temperature $\left(24-26^{\circ}\right)$ was the hypophase. The surface area and surface tension were simultaneously plotted by an $\mathrm{X}-\mathrm{Y}$ recorder.

During a single compression, the change in surface tension as the surface area of the balance decreased is related to the surfactant content of the aliquot layered onto the hypophase $(3,16)$. The quantity of surfactant applied to the balance was quantitated by multiplying the surface area at $12 \mathrm{mN} / \mathrm{m}$ surface tension by 0.7 and using the known dilutions $(16,22,24)$. The surface balance measurement had a reproducibility of $\pm 7 \%$ on repeated determinations, and a minimum sensitivity of $6 \mu \mathrm{g}$ surfactant.

After lavage, the lung of each animal was removed, stripped of all visible extrapulmonary tissue, and dried for $24-48 \mathrm{hr}$ at $50^{\circ}$. In two litters, additional drying resulted in no weight change on reweighing. The mean surfactant per gram of dry lung was determined for each experimental pair.

To validate further our surfactant assay, we prepared 16 additional lavage samples from fetal rabbit pups at 27-31 days of gestation by the method described previously. The surfactant pellet after ultracentrifugation was divided into two portions. One portion was assayed on the surface balance and the other for disaturated phosphatidylcholine (DSPC) after reaction with osmium tetroxide $(9,20)$. A modification of the Bartlett technique (1) was used to quantitate the DSPC phosphorus. Total DSPC was calculated by multiplying the phosphorus result by 25 .

Statistical analysis was done using Student's test for paired or unpaired variables as appropriate. All values are expressed as the mean \pm SEM.

\section{RESULTS}

The surface balance measurement correlates well with paired lung lavage samples assayed for DSPC after osmium tetroxide reaction (20) $(r=0.93 ; P<0.001$, Fig. 1). Since DSPC is the major surface active component of surfactant, this would imply that the surface balance measurement accurately estimates surfactant content of the lavage fluid (3).

\section{RESPIRATORY ACTIVITY AFTER BIRTH}

Because gasping within the amniotic sac may alter surfactant recovery we carefully observed activity of the pups after delivery. The control (nonbreathing) pups responded tu the injection by struggling. After this stimulus, few pups were active and single respiratory efforts were only infrequently observed.

The air-breathing pups always developed a eupneic respiratory pattern. The pups subjected to $10-15 \% \mathrm{O}_{2}$ were cyanotic and inactive, but maintained hypernea throughout the breathing period. The nitrogen-breathing pups, whether or not pretreated with atropine, and the tracheal occlusion pups maintained gasping respirations throughout the $30 \mathrm{~min}$. Both of these groups gasped spontaneously $1-2 \mathrm{times} / \mathrm{min}$ for the first $20 \mathrm{~min}$. Generally, during the final $10 \mathrm{~min}$, these pups required mild stimulation to initiate gasps. Despite progressive acidosis, hypercarbia, and hy- poxia $(13,19)$, these animals would have been capable of autoresuscitation throughout the period of gasping $(11,13)$. At the end of $30 \mathrm{~min}$, these pups were responsive to cutaneous stimuli.

\section{PULMONARY SURFACTANT AFTER BIRTH}

Compared to their littermate controls, the surfactant recovered by pulmonary lavage of the air-breathing pups was increased (Fig. 2A). The mean alveolar surfactant content in the pulmonary lavage of the control pups was $1.40 \pm 0.22 \mathrm{mg} / \mathrm{g}$ dry lung ( \pm SEM). The lavage surfactant from the air-breathing pups of 1.85 $\pm 0.19 \mathrm{mg} / \mathrm{g}$ dry lungs was significnatly higher $(n=11$ litters; paired $t$ test, $P<0.01$ ).

When compared with their littermate controls, the nonpretreated nitrogen-breathing pups showed a significant increase of surfactant in the pulmonary lavage (from $1.61 \pm 0.35 \mathrm{mg} / \mathrm{dry}$ lung in littermate controls to $2.41 \pm 0.58$ in the $\mathrm{N}_{2}$ breathing pups; $P$ $<0.03, n=6$ litters; Fig. 2B). However, the surfactant content in pulmonary lavage fluid from the pups with tracheal occlusion did not increase $(1.34 \pm 0.33 \mathrm{mg} / \mathrm{dry}$ lung in littermate controls $v s$. $1.41 \pm 0.28$ in the occluded pups; $n=7$ litters). Hence, without gas lung expansion, the amount of surfactant recoverable by pulmonary lavage did not increase during asphyxia (Fiz. 2C).

Figure 3 demonstrates that the lavage surfactant content of the atropine pretreated pups subjected to the $100 \%$ nitrogen and/or hypoxic environment ( $1.89 \pm 0.25 \mathrm{mg} /$ dry lung) was unchanged

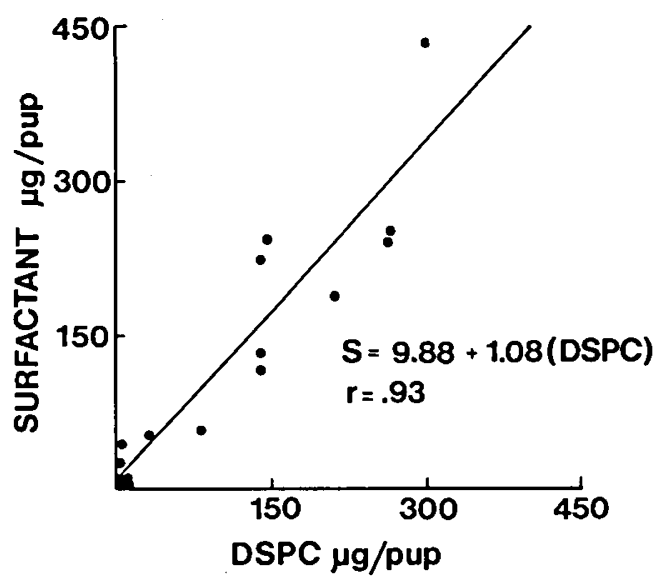

Fig. 1. Results of 16 fetal lung lavage samples assayed for surfactant (S) with the surface balance and disaturated phosphatidylcholine (DSPC). The linear correlation is significant $(P<0.001)$.
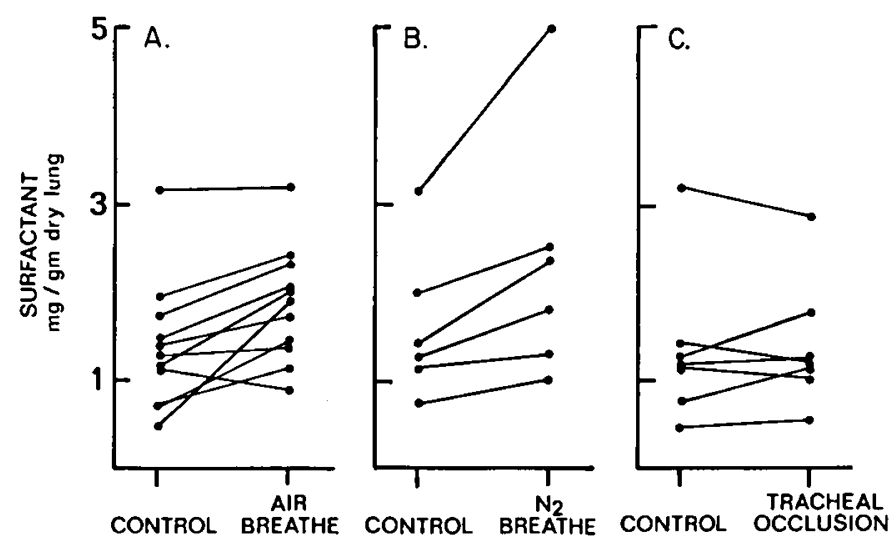

Fig. 2. The effect of A. air-breathing; B. nitrogen-breathing; and C. tracheal occulusion on recovered pulmonary surfactant. The lines connect fetal control and experimental littermates. Surfactant is significantly increased during air-breathing $(n=11$ litters, $P<0.01)$ and nitrogenbreathing ( $n=6$ litters, $P<0.03)$, but not after tracheal occlusion $(n=7$ litters). 


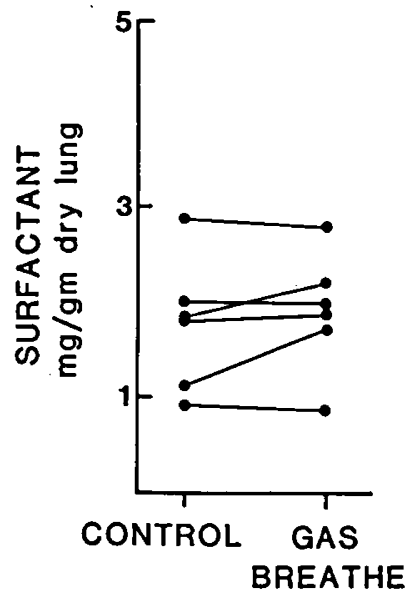

Fig. 3. The effect of maternal pretreatment with atropine on recovered fetal pulmonary surfactant. The lines connect littermate control pups and pups subjected to either hypoxia and/or anoxia ( $n=6$ litters, difference not significant).

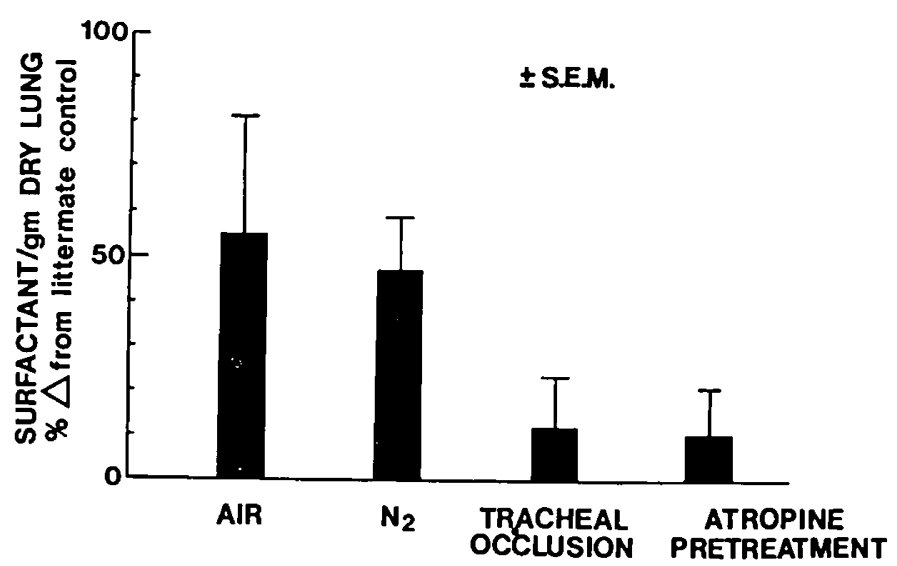

Fig. 4. Mean percent increase of recovered surfactant from the littermate controls for each experimental group. The difference between the nitrogen-breathing and tracheal occlusion pups is significant $(P<0.05)$. Similarly, the difference between atropine pretreated gas-breathing pups, and nonpretreated control pups subjected to similar levels of hypoxia and/ or anoxia (not shown) is significant $(P<0.03)$.

compared with their littermate controls $(1.77 \pm 0.29)$. In comparison, the surfactant levels of the nonatropine pretreated nitrogen or $10-15 \% \quad \mathrm{O}_{2}$-breathing pups were significantly greater $(P<$ $0.003)$ than their nonbreathing littermates $(2.59 \pm 0.40 v s .1 .79 \pm$ $0.29 ; n=9$ litters). The pretreated and nonpretreated control pups did not differ in dry lung weight $(0.143 \pm 0.007$ vs. $0.139 \pm 0.004$ $\mathrm{g})$, maximal lung volume $(2.70 \pm 0.10 v s .2 .63 \pm 0.07 \mathrm{ml})$, and the percent of maximal lung volume remaining on deflation to $10 \mathrm{~cm}$ $\mathrm{H}_{2} \mathrm{O}$ transpulmonary pressure $(73.0 \pm 2.9$ vs. $73.1 \pm 1.6 \%)$.

Figure 4 summarizes the data as percent change of the lavage surfactant content from the littermate control level. The percent increase from control was the same for nonpretreated air and nitrogen-breathing pups, but a significant differnce occurred between the nonpretreated nitrogen-breathing and the tracheal occlusion pups (unpaired $t$ test, $P<0.05, n=13$ ). Similarly, a significant difference $(P<0.03)$ was found in the percent surfactant increase of the nonpretreated pups when compared with the change that occurred in the atropine pretreated pups.

\section{DISCUSSION}

Four reports have shown that exogenous catechols augment surfactant secretion in the fetus $(6,7,18,30)$. Asphyxia causes a generalized sympathetic discharge as well as the release of adrenal catecholamines and possibly other neurohumoral agents (4). The experiments with anoxic pups were designed to differentiate the effects of endogenous neurohumoral agents or other events secondary to asphyxia from the effects of expansion of the lungs at birth. Because both the nitrogen-breathing and occluded pups never breathed room air, they were presumed to be severely hypoxic throughout the period after birth. During gasping, $\mathrm{PO}_{2}$, $\mathrm{PCO}_{2}$ and $\mathrm{pH}$ of nonventilating and nitrogen-ventilating animals has been shown to be similar (13). The nitrogen-breathing pups experienced both asphyxia and pulmonary gas expansion during the 30 -min period of respiratory activity. When compared with their littermate controls, the nitrogen-breathing pups showed a significant increase in surfactant recovered by pulmonary lavage. On the other hand, no change in surfactant over control values was observed in the occluded pups who, though also stressed by asphyxia, did not expand their lungs with gas. The respiratory efforts of these pups were presumably isometric. Because the percent increase of alveolar surfactant in the nitrogen-breathing pups was equivalent to the increase observed in the air-breathing pups, and because the lavage surfactant of the occluded pups did not change significantly from the control amount, we conclude that inflation of the lungs with gas is a major factor in producing increased alveolar surfactant immediately after birth (Fig. 4).

An alternative explanation for our observations is that changes in lung volume or lung water may have led to inaccurate estimations of alveolar surfactant concentration due to differing lavage surfactant recovery between breathing and nonbreathing groups. We discounted this possibility for the following reasons: first, both the control and gas-breathing pups were inflated to maximun lung volume and then deflated to atmospheric pressure. The pressure volume relationship on deflation from maximal lung capacity of 29.5-day control pups is the same as that of air-breathing littermates (5). Thus, despite differences in alveolar surfactant content, on deflation to atmospheric pressure, the lung volume of the control, occluded, and air- or nitrogen-breathing pups was presumably similar at the onset of pulmonary lavage. Second, the failure to find a significant difference between atropine pretreated control and breathing pups indicates that breathing per se does not affect surfactant recovery by lavage. Furthermore, Gluck et al. (12) have demonstrated that the pulmonary lavage of airbreathing pups contained increased percent surface-active lecithin compared with nonbreathing controls. This finding specifically indicates increased surfactant secretion rather than a nonspecific effect of breathing on lavage efficiency. Fourth, unlike that of the control pups, alveolar water would be reabsorbed in breathing pups. The possibility of surfactant dilution in nonexpanded pups was avoided by the method of concentrating surfactant recovered by the lavage. Lung water may have decreased in the occluded pups (27), however, because no difference in surfactant content was noted when compared with control littermates, this possibility is of negligible importance.

It is possible that the neck ligature significantly damaged neck neural structures leading to reduced surfactant secretion. Because the pups were capable of gasping and were responsive to tactile stimulation suggests that the phrenic nerves and spinal cord are not damaged and, therefore, we infer that other nerves contained within the neck would not be damaged. It is unlikely that neck ligature would have interfered with an adrenergic influence on surfactant secretion. In the rabbit pup, cardiac sympathetic innervation is delayed until after birth (10), suggesting that direct pulmonary sympathetic innervation may also be delayed. Thus, the adrenal medulla would be the major source of increased catecholamines. Stimulation of the adrenal medulla may occur either through the direct action of anoxia or through the splanchnic nerves (4). Therefore, the major source of increased catechols in the rabbit pups would not be affected by the neck ligature.

Bilateral vagotomy leads to reduced osmiophilic bodies in alveolar type II cells suggesting that the vagus plays a role in surfactant secretion $(2,15,17)$. Pilocarpine, a cholinergic agonist, has been shown by several investigators to stimulate surfactant 
secretion $(5,14,21)$. Smith (28) has shown that cultured cells similar to type II alveolar cells secrete increased amounts of phosphatidylcholine in response to acetylcholine and this effect may be blocked by atropine. In adult rabbits, increased pulmonary ventilation leads to increased alveolar surfactant and this effect is mediated at least partially by the vagus nerve (23). In the present experiments, atropine blocked the effect of gas ventilation on net accumulation of alveolar surfactant after birth. Thus, we suggest that, at birth, gas expansion of the lung augments surfactant secretion from type II alveolar cells and this effect is mediated via a cholinergic mechanism (i.e., the vagus nerve).

We have not excluded the possibility that adrenergic mechanisms also lead to increased alveolar surfactant $(5,6,17,18,30)$. However, cholinergic transmission at the sympathetic ganglia is primarily via nicontinic receptors and, except in high dosage, atropine does not block these receptors. Therefore, it is unlikely that under the present experimental conditions an adrenergic mechanism is the primary effector of increased alveolar surfactant. We speculate that a vagal mechanism may stimulate secretion of intracellular surfactant stores in response to lung expansion. Alternatively, adrenergic mechanisms may lead indirectly to increased alveolar surfactant by an effect on intracellular synthesis which is followed by increased surfactant secretion. This latter mechanism would provide a slower, less precise control of alveolar surfactant and may require a longer stimulus duration to be recognized.

\section{CONCLUSION}

Surfactant recovered by alveolar lavage of 30-day-gestation rabbit pups increased after gas ventilation when compared with nonbreathing controls. This effect was blocked by atropine suggesting that a cholinergic mechanism is responsible for the increase.

\section{REFERENCES AND NOTES}

1. Bartlett, G. R.: Phosphorus assay in column chromatography. J. Biol. Chem., 234: 466 (1959).

2. Bolande, R. P., and Klaus, M. H. L.: The morphologic demonstration of an alveolar lining layer and its relationship to pulmonary surfactant. Am. J. Pathol., 45: 449 (1964).

3. Clements, J. A., Nellenbogen, J., and Trahan, H. J.: Pulmonary surfactant and the evolution of the lung. Science, 169: 603 (1970).

4. Comline, R. S., and Silver, M.: Development of activity in the adrenal medulla of the fetus and the newborn animal. Br. Med. Bull, 22: 16 (1966).

5. Corbet, A. J. S., Flax, P., and Rudolph, A. J.: Reduced surface tension in lungs of fetal rabbits injected with pilocarpine. J. Applied Physiol., 4I: 7 (1976).

6. Corbet, A. J. S., Flax, P., and Rudolph, A. J.: Role of autonomic nervous system controlling surface tension in fetal rabbit lungs. J. Appl. Physiol., 43: 1039 (1977).

7. Enhorning, G., Chamberlain, D., Contreras, C., Burgoyne, R., Robertson, B.: Isoxuprine induced release of pulmonary surfactant in the rabbit fetus. Am. J. Obstet. Gynecol., 129: 197 (1977).

8. Faridy, E. E.: Effect of distension in excised dog's lungs. Resp. Physiol, 27: 99 (1976).

9. Folch, J., Lees, M., Sloan Stanley, G. H. L.: A simple method for the isolation and purification of total lipides from animal tissues. J. Biol. Chem., 226: 497 (1957).

10. Friedman, W. F., Pool, P. W., Jacobowitz, D., Seagreen, S. C., and Braunwald, E.: Sympathetic innervation of the developing rabbit heart. Biochemical and histochemical comparisons of fetal, neonatal, and adult myocardium. Circ. Res., 23: 25 (1968).

11. Glass, H. G., Sayder, F. F., and Webster, E.: The rate of decline in resistance to anoxia of rabbits, dogs and guinea pigs from the onset of viability to adult life. Am. J. Physiol., 140: 609 (1944).

12. Gluck, L. Motoyama, E. K., Smits, H. L., and Kulovich, M. V.: The biochemical development of surface activity in mammalian lung. I. The surface active phospholipids; the separation and distribution of surface-active lecithin in the lung of the developing fetus. Pediatr Res., 1: 237 (1967).

13. Godfrey, S.: Bloodgases during asphyxia and resuscitation of fetal and newborn rabbits. Resp. Physiol., 4: 309 (1968).

14. Goldenberg, V. E., Buckingham, S., and Sommers, S. C.: Pilocarpine stimulation of granular pneumocyte secretion. Lab. Invest., 20: 147 (1969).

15. Goldenberg, V. E., Buckingham, S., and Sommers, S. C.: Pulmonary alveolar lesions in vagotomized rabbits. Lab. Invest., 16: 693 (1967).

16. King, R. J., and Clements, J. A.: Surface active materials from dog lung. I. Method of isolation. Am. J. Physiol., 223: 707 (1972).

17. Klaus, M., Reiss, O. K., Tooley, W. H., Piel, C., and Clements, J. A.: Alveolar epithelial cell mitochondria as source of the surface-active lung lining. Science, 137: 750 (1962).

18. Lawson, E. E., Brown, E. B., Torday, J. S., Madansky, D. L., and Taeusch, H. W., Jr.: The effect of epinephrine on tracheal fluid flow and surfactant efflux in fetal sheep. Am. Rev. Resp. Dis., 118: 1023 (1978).

19. Lawson, E. E., and Thach, B. T.: Respiratory patterns during progressive asphyxia in newborn rabbits. J. Applied Physiol.: Respir. Environ. Exercise Physiol., 43: 468 (1977).

20. Mason, R. J., Nellenbogen, J., and Clements, J. A.: Isolation of disaturated phosphatidylcholine with osmium tetroxide. J. Lipid Res., 17: 281 (1976).

21. Massaro, D.: In vivo protein secretion by lung. Evidence for active secretion and interspecies differences. J. Clin. Invest., 56: 263 (1975).

22. Mescher, E. J., Platzker, A. C. G., Ballard, P. L., Kitterman, J. A., Clements, J. A., and Tooley, W. H.: Ontogeny of tracheal fluid, pulmonary surfactant, and plasma corticoids in the fetal lamb. J. Applied Physiol., 39: 1017 (1975).

23. Oyarzun, M. J., and Clements, J. A.: Ventilatory and cholinergic control of pulmonary surfactant in the rabbit. J. Appl. Physiol.: Respir. Environ. Exercise Physiol., 43: 39 (1977).

24. Platzker, A. C. G., Kitterman, J. A., Mescher, E. J., Clements, J. A., and Tooley, W. H.: Surfactant in the lung and tracheal fluid of the fetal lamb and the acceleration of its appearance by dexamethasone. Pediatrics, 56: 554 (1975).

25. Rooney, S. C., Gobran, L. I., and Wai-Lee, T. S.: Stimulation of surfactant production by oxytocin-induced labor in the rabbit. J. Clin. Invest., 60: 754 (1977).

26. Sawin, P. B., and Glick, D.: Atropinesterase, A genetically determined enzyme in the rabbit. Proc. Nat. Acad. Sci., 29: 55 (1943).

27. Scarpelli, E. M., Condorelli, S., and Cosmi, E. V.: Lamb fetal pulmonary fluid. 1. Validation and significance of method for determination of volume and volume change. Pediatr. Res., 9: 190 (1975).

28. Smith, B. T.: Cell line A549: a model system for the study of alveolar type II cell function. Am. Rev. Resp. Dis., 115: 285 (1977).

29. Taeusch, H. W. Jr., Wyszogrodski, I., Wang. N. S., and Avery, M. E.: Pulmonary pressure-volume relationships in premature fetal and newborn rabbits. J. Appl. Physiol., 37: 809 (1974).

30. Wyszogrodski, I., Taeusch, H. W., Jr., and Avery, M. E.: Isoxuprine-induced alterations of pulmonary pressure-volume relationships in premature rabbits. Am. J. Obstet. Gynecol., 119: 1107 (1974).

31. This research was supported in part, by the National Institute of Health, research grant HD8529 from the National Institute of Child Health and Development.

32. Dr. Lawson is a Training Fellow of the American Lung Association.

33. Requests for reprints should be addressed to: Dr. Edward E. Lawson, Department of Pediatrics, 509 Burnett-Womack Clinical Sciences Building, University of North Carolina, Chapel Hill, NC 27514 (USA).

34. Received for publication February $9,1978$.

35. Accepted for publication May 26, 1978. 